2

3 16 9

\title{
Genomic inference of a human super bottleneck in Mid-Pleistocene transition
}

$$
\begin{gathered}
\text { Wangjie } \mathrm{Hu}^{1 \dagger} \text {, Ziqian } \mathrm{Hao}^{1 \dagger} \text {, Pengyuan } \mathrm{Du}^{1} \text {, Fabio Di Vincenzo }{ }^{3} \text {, Giorgio Manzi } \\
\\
\text { Yi-Hsuan } \mathrm{Pan}^{2 *} \text {, Haipeng } \mathrm{Li}^{1,5,6^{*}}
\end{gathered}
$$

${ }^{1}$ CAS Key Laboratory of Computational Biology, Shanghai Institute of Nutrition and Health, University of Chinese Academy of Sciences, Chinese Academy of Sciences; Shanghai 200031, China.

${ }^{2}$ Key Laboratory of Brain Functional Genomics of Ministry of Education, School of Life Science, East China Normal University; Shanghai 200062, China.

${ }^{3}$ Natural History Museum, University of Florence; Florence, Italy.

${ }^{4}$ Department of Environmental Biology, Sapienza University of Rome; Italy.

${ }^{5}$ Center for Excellence in Animal Evolution and Genetics, Chinese Academy of 4 Sciences; Kunming 650223, China.

${ }^{6}$ Lead Contact

7 *Corresponding Authors: yxpan@ @ sat.ecnu.edu.cn; lihaipeng@picb.ac.cn

$8 \dagger$ These authors contributed equally to this work. 


\section{SUMMARY}

The demographic history is a foundation of human evolutionary studies. However, the ancient demographic history during the Mid-Pleistocene is poorly investigated while it is essential for understanding the early origin of humankind. Here we present the fast infinitesimal time coalescent (FitCoal) process, which allows the analytical calculation of the composite likelihood of a site frequency spectrum and provides the precise inference of demographic history. We apply it to analyze 3,154 present-day human genomic sequences. We find that African populations have passed through a population super bottleneck, a small effective size of approximately 1,280 breeding individuals between 930 and 813 thousand years ago. Further analyses confirm the existence of the super bottleneck on non-African populations although it cannot be directly inferred. This observation, together with simulation results, indicates that confounding factors, such as population structure and selection, are unlikely to affect the inference of the super bottleneck. The time interval of the super bottleneck coincides with a gap in the human fossil record in Africa and possibly marks the origin of Homo heidelbergensis. Our results provide new insights into human evolution during the Mid-Pleistocene.

\section{Keywords}

Demographic history inference, FitCoal, site frequency spectrum, population bottleneck, Mid-Pleistocene transition 


\section{INTRODUCTION}

With African hominid fossils, the origin of anatomically modern humans has been determined to be approximately 200 thousand years (kyr) ago (White et al., 2003). Based on present-day human genomes, the recent demographic history of humans has been intensively studied which reveals the world-wide spread of our ancestors (Li and Durbin, 2011; Liu and Fu, 2015; Manica et al., 2007; Nielsen et al., 2017; Ramachandran et al., 2005; Stoneking and Krause, 2011; Terhorst et al., 2017). However, the ancient demographic history during the Mid-Pleistocene is still poorly investigated while it is essential for understanding the early origin of humankind. It is mainly due to limitations of existed methods since this task requires a precise estimate for the ancient demographic history. Thus a novel approach is needed to improve the inference accuracy of demographic history.

As site frequency spectrum (SFS) plays an essential role in demographic inference (Excoffier et al., 2013; Griffiths and Tavaré, 1996; Gutenkunst et al., 2009; Li and Stephan, 2006; Liu and Fu, 2020; Liu and Fu, 2015; Terhorst et al., 2017), many efforts have been made to derive its analytical formula under a predefined demographic model (Fu, 1995; Jouganous et al., 2017; Zivković and Wiehe, 2008). Therefore, to precisely infer recent and ancient demography, we developed the fast infinitesimal time coalescent (FitCoal) process (Figure 1) that analytically derives expected branch length for each SFS type under arbitrary demographic models. It is effective for a wide range of sample sizes in the analytical calculation of the composite likelihood of a given SFS. FitCoal first maximizes the likelihood with the constant size model and then increases the number of inference time intervals and re-maximizes the likelihood until the best model is found. FitCoal does not need prior information on demography, and its accuracy is confirmed by simulation. The demographic inference of FitCoal is more precise than that of PSMC ( $\mathrm{Li}$ and Durbin, 2011) and stairway plot ( $\mathrm{Liu}$ and $\mathrm{Fu}, 2015$ ), and the effects of positive selection and sequencing error can be easily excluded.

We then used FitCoal to analyze large sets of present-day human genomic 
sequences sampled from 10 African and 40 non-African populations. The inferred recent demographic histories, including recent population size expansion/reduction and the out-of-African bottleneck, are consistent with previous studies (Altshuler et al., 2015; Bergstrom et al., 2020; Li and Durbin, 2011; Prugnolle et al., 2005; Ramachandran et al., 2005; Schiffels and Durbin, 2014; Terhorst et al., 2017). However, we found that our ancestors experienced a super bottleneck and the effective size of our ancestors remained small (about 1,280 breeding individuals) between 930 and 813 thousand years ago. The super bottleneck was directly inferred on African populations but only indirectly detected on non-African populations, which is expected by the coalescent theory. This observation, together with simulation results, indicates that confounding factors, such as population structure and selection, are unlikely to affect the inference of the super bottleneck during the Mid-Pleistocene. The super bottleneck not only explains a gap of the human fossil record in Africa between roughly 900 and $600 \mathrm{kyr}$ ago (Profico et al., 2016), but also may represent a major transition in human evolution, possibly leading to the origin of $H$. heidelbergensis: the alleged ancestral species of modern humans (Profico et al., 2016; Stringer, 2016).

\section{RESULTS}

\section{Fast Infinitesimal Time Coalescent Process}

As analytical result of expected branch length for each SFS type is essential for theoretical population genetics and demographic inference (Excoffier et al., 2013; Fu, 1995; Li and Stephan, 2006; Zivković and Wiehe, 2008), we developed the fast infinitesimal time coalescent (FitCoal) process to accomplish the task (Figure 1). The analytical result of expected branch length for each SFS type was presented in the STAR $\star$ METHODS. For FitCoal calculation, each of millions of time intervals $\Delta t$ was set extremely small, and the population size was assumed to be constant within each infinitesimal time interval. The probabilities of all states were calculated backward in time. During each $\Delta \mathrm{t}$, the branches were categorized according to their state. For each state, the branch length was multiplied by its probability and population size and then transformed to calculate the expected branch length of each 
SFS type. Because the expected branch length of a SFS type is equal to the sum of the expected branch length of this type during each time interval, the latter can be rescaled and tabulated, making the calculation of the expected branch lengths extremely fast under arbitrary demographic histories. Hereafter, tabulated FitCoal is referred to as FitCoal for short, unless otherwise indicated.

\section{FitCoal Demographic Inference}

After the expected branch lengths were obtained, the composite likelihood of the SFS observed in a sample was calculated (Excoffier et al., 2013; Hudson, 2001; Li and Stephan, 2006; Liu and Fu, 2015). As each single nucleotide polymorphism (SNP) was treated independently, FitCoal did not need phased haplotype data. When inferring demography, the likelihood was maximized in a wide range of demographic scenarios. The FitCoal likelihood surface is smooth (Figure S1), so it is efficient to maximize the likelihood. FitCoal considered both instantaneous populations size changes (Li and Durbin, 2011; Liu and Fu, 2015; Schiffels and Durbin, 2014) and long-term exponential changes of population in order to generate various demographic scenarios.

\section{Demographic Inference on Simulated Data}

The accuracy of FitCoal was validated by simulation and comparing its demographic inferences with those of PSMC (Li and Durbin, 2011) and stairway plot (Liu and Fu, 2015) (Figure 2). Six demographic models, examined in the former study (Liu and Fu, 2015), were considered by simulating 200 independent data sets under each model. The medians and 95\% confidence intervals of demography were then determined by FitCoal with the assumption that a generation time is 24 years (Liu and $\mathrm{Fu}, 2015$; Scally and Durbin, 2012) and the mutation rate is $1.2 \times 10^{-8}$ per site per generation for human populations (Campbell et al., 2012; Conrad et al., 2011; Kong et al., 2012; Liu and Fu, 2015).

FitCoal was found to precisely infer demographic histories (Figure 2). In general, the confidence intervals of FitCoal-inferred histories were narrower than those of PSMC and stairway plot, indicating a better FitCoal-demographic inference. The inference accuracy can be improved by increasing sample size and length of sequence (Figure S2). Our results confirmed that SFS allows precise recovery of the 
demographic history (Bhaskar and Song, 2014). The proportion of the most recent change type inferred from six models above showed that FitCoal can distinguish instantaneous and exponential changes (Table S1).

Since a demographic event may affect every SFS type, demographic history can be inferred using a subset of SFS. Results of simulation confirmed that FitCoal accurately determined demographic history based on truncated SFSs (Figures S3 and S4), thus reducing the impact of other factors, such as positive selection (Figure S5) and sequencing error, on FitCoal analysis.

\section{Demographic Inference of African Populations}

To infer the demographic histories of African populations, seven African populations in the 1000 Genomes Project (1000GP) (Altshuler et al., 2015) were analyzed by FitCoal. Only non-coding regions, defined by GENCODE (Frankish et al., 2019), were used in order to avoid the effect of purifying selection. To avoid the potential effect of positive selection (Fay and $\mathrm{Wu}, 2000$ ), high-frequency mutations were excluded from the analysis.

Results showed that all seven African populations passed through a super bottleneck around $914(854-1,003)$ kyr ago and that this bottleneck was relieved about 793 (772-815) kyr ago (Figures 3A-C and S6; Table S2). The average effective population size of African populations during the bottleneck period was determined to be 1,270 (770-2,030). Although traces of the bottleneck were observed in previous studies, the bottleneck was ignored because its signatures were too weak to be noticed (Altshuler et al., 2015; Bergstrom et al., 2020; Li and Durbin, 2011; Schiffels and Durbin, 2014; Terhorst et al., 2017). After the bottleneck was relieved, the population size was increased to $27,080(25,300-29,180)$, a 20 -fold increase, around $800 \mathrm{kyr}$ ago. This population size remained relatively constant until the recent expansion.

To avoid the potential effects of low sequencing depth $(\sim 5 \mathrm{x})$ of non-coding regions in the 1000GP on the analysis, the autosomal non-coding genomic polymorphism of Human Genome Diversity Project - Centre d'Etude du Polymorphisme Humain panel (HGDP-CEPH) with high sequencing coverage ( $35 \mathrm{x})$ was analyzed (Bergstrom et al., 2020). Populations with more than 15 individuals each were examined. Results showed that the super bottleneck occurred on all three African populations in HGDP-CEPH between 1,257 (1,042-1,527) and 859 (856-864) 
kyr ago (Figures 3D-F and S7; Table S3), and the average population size during the bottleneck period was $1,300(908-1,670)$. This number was very similar to that $(1,270)$ estimated from the data of 1000GP.

After the bottleneck was relieved, the population sizes of the two HGDP-CEPH agriculturalist populations were increased to 27,300 and 27,570 (Figures 3E and S7; Table S3), consistent with the 1000GP estimate of 27,280. The Biaka, a hunter-gatherer population, had a larger population size of 35,330, suggesting a deep divergence between this and other agriculturalist populations (Hsieh et al., 2016; Schlebusch and Jakobsson, 2018; Skoglund et al., 2017). The Biaka population was found to have a recent population decline (Figures 3D and S7), as previously observed (Bergstrom et al., 2020). These results suggest that hunter-gatherer populations were widely spread and decreased when agriculturalist populations were expanded.

To provide a precise inference of the super bottleneck, the results from the two data sets were combined. After analyzing the inferred time of instantaneous change of 10 populations, the super bottleneck was inferred to last for about 117,000 years, from 930 (854-1,042; s.e.m.: 23.52) to 813 (772-864; s.e.m.: 11.02) kyr ago. The effective size during the bottleneck period was precisely determined to be 1,280 (767-2,031; s.e.m.: 131). A loss of $65.85 \%$ in current genetic diversity of human populations was estimated because of the bottleneck.

\section{Demographic Inference of Non-African Populations}

No super bottleneck was directly observed on all 19 non-African populations in 1000GP (Figures 3A-C and S6; Table S4). The ancestral population size of these populations was determined to be $20,260(18,850-22,220)$, similar to that determined in previous studies (Bergstrom et al., 2020; Li and Durbin, 2011; Schiffels and Durbin, 2014; Terhorst et al., 2017). The population size of 1000GP non-African populations started to decline around 368 (175-756) kyr ago, suggesting that African and non-African divergence occurred much earlier than the out-of-Africa migration (Altshuler et al., 2015; Bergstrom et al., 2020; Li and Durbin, 2011; Nielsen et al., 2017; Schiffels and Durbin, 2014; Terhorst et al., 2017). European and South Asian populations were found to have a relatively weaker out-of-Africa bottleneck than East Asian populations, and the bottleneck severity was found to correlate with their geographic distance to Africa, consistent with the observed correlation between 
heterozygosity and geographic distance (Prugnolle et al., 2005; Ramachandran et al., 2005). A weak bottleneck was observed on American populations, probably because of recent admixture (Altshuler et al., 2015). All 1000GP non-African populations were found to increase in size recently.

The super bottleneck was also not directly detected in all 21 HGDP-CEPH non-African populations (Figures 3D-F and S7; Table S5). The ancestral population size of these populations was determined to be $20,030(19,060-21,850)$, very similar to that $(20,260)$ estimated from 1000GP. These populations started to decline 367 (167-628) kyr ago. A positive correlation was also observed between the severity of out-of-Africa bottleneck and their geographic distance to Africa. The Middle East populations had the weakest bottleneck, while the Maya, an American population, had the strongest bottleneck. Similar to 1000GP non-African populations, most HGDP-CEPH non-African populations were found to increase in size recently, except an isolated Kalash population, consistent with previous studies (Ayub et al., 2015; Bergstrom et al., 2020).

\section{Super Bottleneck in the Early Middle Pleistocene}

The super bottleneck was directly inferred on all 10 African populations, but not on all 40 non-African populations. To investigate this observation, simulations were performed with three 1000GP demographic models, designated Bottleneck I, II, and III (Figure 4). Bottleneck I simulated the average inferred demographic history of African populations with the super bottleneck, and Bottleneck II and III simulated the demography of non-African populations without and with the super bottleneck. Both Bottleneck I and II were inferred correctly in all simulated data sets (Table S6). However, no super bottleneck was detected in Bottleneck III simulations. The super bottleneck was found to cause a population size gap between the true model and inferred demographic history after the bottleneck was relieved, suggesting a hidden effect of the super bottleneck on non-African populations. Simulations were then extended to HGDP-CEHP populations with Bottleneck models IV-VI, and similar results were obtained (Figure S8; Table S7). When simulations were performed on three artificial models (Bottleneck VII-IX) with various demographic parameters, the population size gap was still detected (Figure S9; Table S8). These results suggest a hidden effect of the super bottleneck on non-African populations. 
The population size gap was found in both 1000GP and HGDP-CEPH data sets (Figure 3A, D). After the bottleneck was relieved, the average population sizes of non-African populations were determined to be 20,260 and 20,030, respectively, while those of African agriculturalist populations were 27,080 and 27,440, respectively in these two data sets. The observed population size gap was 7,020, probably due to the hidden effect of the super bottleneck on non-African populations.

The reasons were then investigated why the super bottleneck had different effects on African and non-African populations. Results showed that non-African populations had the out-of-Africa bottleneck, but African populations lacked such bottleneck. Therefore, the standard coalescent time of non-African populations was larger than that of African populations (Figure 3C, F). As African populations had more coalescent events occurred during the bottleneck period, the bottleneck was more readily inferred. The mathematical proof on this issue was described in the STAR $\star$ METHODS.

\section{DISCUSSION}

In this study, we develop FitCoal, a novel model-flexible method for demographic inference. One key characteristic feature of FitCoal is that the analytical result of expected branch length is obtained for each SFS type under arbitrary demographic models. This enables us to calculate precisely the likelihood. Second, the tabulated FitCoal is used to calculate rapidly the likelihood, making FitCoal economical of inference time. Third, the confounding effects of sequencing error and positive selection can be easily avoided by discarding rare and high-frequency mutations without losing inference accuracy. Fourth, exponential change is allowed within each inference time interval which represents a long-term continuous population change. This feature provides a better approximation to the demographic history of real populations while PSMC (Li and Durbin, 2011) and stairway plot (Liu and $\mathrm{Fu}, 2015)$ need multiple instantaneous changes to fit an exponential change. Last but not least, inference time intervals are variable during the demographic inference, leading to a better inference of ancient demographic events. Since coalescent events become rare when tracing backward in time, the length of time interval is usually set to increase progressively ( $\mathrm{Li}$ and Durbin, 2011; Liu and Fu, 2015; Schiffels and Durbin, 2014; Terhorst et al., 2017). Although this strategy can capture recent 
demographic events, it may miss ancient ones. Therefore, FitCoal can make a fast and accurate inference for recent and ancient demographic events.

The most important discovery with FitCoal in this study is that human ancestors passed through a super bottleneck during the Mid-Pleistocene. Strikingly, the super bottleneck is inferred on all the 10 African populations while only a hidden effect of the super bottleneck is detected on all the 40 non-African populations. This observation is not only explained by the coalescent theory (see the section above) but also exclude the possibility that the super bottleneck is falsely inferred due to positive selection, population structure, sequencing error, and other confounding factors. If the inferred demographic histories of non-African populations are affected by those confounding factors, the super bottleneck should be falsely inferred on non-African populations. Moreover, large-scale simulations demonstrate that FitCoal did not falsely infer a bottleneck due to the existence of positive selection (Figure S5) and population structure (Figures S35 and S36) in African populations. Therefore, the super bottleneck exists during the Mid-Pleistocene and is shared by African and non-African populations.

The ancient population size reduction around $930 \mathrm{kyr}$ ago was likely to be driven by the climatic changes at the transition between the Early and Middle Pleistocene (Lisiecki and Raymo, 2005). During the transition, low-amplitude $41 \mathrm{kyr}$ obliquity-dominated glacial cycles shifted to quasi-periodic, low frequency $100 \mathrm{kyr}$ periodicity, and climate change became more extreme and unpredictably associated with a longer dry period in Africa and a large faunal turnover in Africa and Eurasia (Head et al., 2008). Coinciding with this date, archaic humans referable to African Homo erectus became extinct. Subsequently, from about 900 until 600 kyr ago, there is a gap in the human fossil record in Africa (Figure S10) (Profico et al., 2016). Only few fossil specimens have been found in this time span, such as the cranial fragments from Gombore in Ethiopia and the mandibles from Tighenif in Algeria, all of which show features linked to later $H$. heidelbergensis representatives and represent the evolutionary origin of this species (Stringer, 2016). As a matter of fact, our data suggest that the ancestors of modern humans had a very small effective size of 
approximately 1,280 breeding individuals during the bottleneck period. This number is comparable in the same magnitude in the effective size of mammals threatened by extinction ( $\mathrm{Li}$ et al., 2016).

A rapid population recovery was inferred on all 10 African populations with a 20 -fold population growth during a short time period around $813 \mathrm{kyr}$ ago. The earliest archaeological evidence for human control of fire was found in Israel 790 kyr ago (Goren-Inbar et al., 2004). As the control of fire profoundly affected social evolution (Foley and Gamble, 2009) and brain size (Melchionna et al., 2020), it may be associated with the big bang in population size at the end of the super bottleneck. However, climatic changes, as the alternative hypothesis, cannot be ruled out. Thus, the driving force of the rapid population recovery needs to be further studied.

The super bottleneck, which started about one million years ago, might represent a speciation event at the origin of $H$. heidelbergensis and should be strongly related to the gap in the African human fossil record. The questions about where the small ancient population dwelt, and how they survived for such a long time, remain to be investigated. Our findings may also shed light on a debate about the divergence time between Neanderthals/Denisovans and modern humans (between 440 and $270 \mathrm{vs}$ 1,007 kyr ago) (Green et al., 2010; Ni et al., 2021; Reich et al., 2010; Shao et al., 2021). The two estimates can be verified by detecting whether ancestors of Neanderthals/Denisovans passed through the super bottleneck. In the future, a more detailed picture of human evolution during the Pleistocene may be revealed because more genomic sequences of present populations and those of archaic hominins as well as more advanced population genomics methods will be available.

\section{ACKNOWLEDGMENTS}

We thank Daniel Zivković for sharing his codes to calculate the expected branch length, and Xiaoming Liu for sharing his simulated results. This work was supported by grants from the Strategic Priority Research Program of the Chinese Academy of Sciences (XDB13040800), the National Natural Science Foundation of China (nos. 31100273, 31172073, 91131010), and National Key Research and Development Project (No. 2020YFC0847000).

\section{AUTHOR CONTRIBUTIONS}


bioRxiv preprint doi: https://doi.org/10.1101/2021.05.16.444351; this version posted June 28, 2021. The copyright holder for this preprint (which was not certified by peer review) is the author/funder, who has granted bioRxiv a license to display the preprint in perpetuity. It is made available under aCC-BY-NC-ND 4.0 International license.

333 W.H., Z.H., Y.H.P., and H.L. conceived and designed the research; W.H., Z.H., and

334 H.L. wrote the code; W.H., Z.H., P.D., F.D.V., G.M., and Y.H.P. analyzed the data;

335 W.H., Z.H., P.D., F.D.V., G.M., Y.H.P., and H.L. wrote the paper.

336

337

DECLARATION OF INTERESTS

338 The authors declare no competing interests. 


\section{REFERENCES}

Altshuler, D.M., Durbin, R.M., Abecasis, G.R., Bentley, D.R., Chakravarti, A., Clark, A.G., Donnelly, P., Eichler, E.E., Flicek, P., Gabriel, S.B., et al. (2015). A global reference for human genetic variation. Nature 526, 68-74.

Ayub, Q., Mezzavilla, M., Pagani, L., Haber, M., Mohyuddin, A., Khaliq, S., Mehdi, S.Q., and Tyler-Smith, C. (2015). The Kalash genetic isolate: ancient divergence, drift, and selection. Am. J. Hum. Genet. 96, 775-783.

Beerli, P. (2004). Effect of unsampled populations on the estimation of population sizes and migration rates between sampled populations. Mol. Ecol. 13, 827-836.

Bergstrom, A., McCarthy, S.A., Hui, R.Y., Almarri, M.A., Ayub, Q., Danecek, P., Chen, Y., Felkel, S., Hallast, P., Kamm, J., et al. (2020). Insights into human genetic variation and population history from 929 diverse genomes. Science 367, eaay5012.

Bhaskar, A., and Song, Y.S. (2014). Descartes' rule of signs and the identifiability of population demographic models from genomic variation data. Ann. Stat. 42, 2469-2493.

Campbell, C.D., Chong, J.X., Malig, M., Ko, A., Dumont, B.L., Han, L., Vives, L., O'Roak, B.J., Sudmant, P.H., Shendure, J., et al. (2012). Estimating the human mutation rate using autozygosity in a founder population. Nat. Genet. 44, 1277-1281.

Chen, G.K., Marjoram, P., and Wall, J.D. (2009). Fast and flexible simulation of DNA sequence data. Genome Res. 19, 136-142.

Chen, H. (2019). A computational approach for modeling the allele frequency spectrum of populations with arbitrarily varying size. Genomics Proteomics Bioinf. 17, 635-644.

Conrad, D.F., Keebler, J.E.M., DePristo, M.A., Lindsay, S.J., Zhang, Y.J., Casals, F., Idaghdour, Y., Hartl, C.L., Torroja, C., Garimella, K.V., et al. (2011). Variation in genome-wide mutation rates within and between human families. Nat. Genet. 43, 712-714.

Durvasula, A., and Sankararaman, S. (2020). Recovering signals of ghost archaic introgression in African populations. Sci. Adv. 6, eaax5097.

Excoffier, L., Dupanloup, I., Huerta-Sanchez, E., Sousa, V.C., and Foll, M. (2013). Robust demographic inference from genomic and SNP data. PLoS Genet. 9, e1003905.

Fay, J.C., and Wu, C.-I. (2000). Hitchhiking under positive Darwinian selection. Genetics 155, 1405-1413.

Foley, R., and Gamble, C. (2009). The ecology of social transitions in human evolution. Philos. Trans. R. Soc. Lond., Ser. B: Biol. Sci. 364, 3267-3279.

Frankish, A., Diekhans, M., Ferreira, A.M., Johnson, R., Jungreis, I., Loveland, J., Mudge, J.M., Sisu, C., Wright, J., Armstrong, J., et al. (2019). GENCODE reference annotation for the human and mouse genomes. Nucleic Acids Res. 47, D766-D773.

Fu, Y.X. (1995). Statistical properties of segregating sites. Theor. Popul. Biol. 48, 172-197. 
400

401

402

403

404

405

406

407

408

409

410

411

412

413

414

415

416

417

418

419

420

421

422

423

Fu, Y.X., and Li, W.H. (1993). Statistical tests of neutrality of mutations. Genetics 133, 693-709.

Goren-Inbar, N., Alperson, N., Kislev, M.E., Simchoni, O., Melamed, Y., Ben-Nun, A., and Werker, E. (2004). Evidence of hominin control of fire at Gesher Benot Ya'aqov, Israel. Science 304, 725-727.

Green, R.E., Krause, J., Briggs, A.W., Maricic, T., Stenzel, U., Kircher, M., Patterson, N., Li, H., Zhai, W., Fritz, M.H.-Y., et al. (2010). A draft sequence of the Neandertal genome. Science 328, 710-722.

Griffiths, R.C., and Tavaré, S. (1996). Monte Carlo inference methods in population genetics. Math. Comput. Modell. 23, 141-158.

Gutenkunst, R.N., Hernandez, R.D., Williamson, S.H., and Bustamante, C.D. (2009). Inferring the joint demographic history of multiple populations from multidimensional SNP frequency data. PLoS Genet. 5, e1000695.

Harpending, H.C., Batzer, M.A., Gurven, M., Jorde, L.B., Rogers, A.R., and Sherry, S.T. (1998). Genetic traces of ancient demography. Proc. Natl. Acad. Sci. USA 95, 1961-1967.

Head, M.J., Pillans, B., and Farquhar, S.A. (2008). The Early-Middle Pleistocene transition: characterization and proposed guide for the defining boundary. Episodes 31, 255.

Hsieh, P., Woerner, A.E., Wall, J.D., Lachance, J., Tishkoff, S.A., Gutenkunst, R.N., and Hammer, M.F. (2016). Model-based analyses of whole-genome data reveal a complex evolutionary history involving archaic introgression in Central African Pygmies. Genome Res. 26, 291-300.

Hudson, R.R. (2001). Two-locus sampling distributions and their application. Genetics 159, 1805-1817.

Hudson, R.R. (2002). Generating samples under a Wright-Fisher neutral model of genetic variation. Bioinformatics 18, 337-338.

Jouganous, J., Long, W., Ragsdale, A.P., and Gravel, S. (2017). Inferring the joint demographic history of multiple populations: beyond the diffusion approximation. Genetics 206, 1549-1567.

Kim, Y., and Stephan, W. (2002). Detecting a local signature of genetic hitchhiking along a recombining chromosome. Genetics 160, 765-777.

Kong, A., Frigge, M.L., Masson, G., Besenbacher, S., Sulem, P., Magnusson, G., Gudjonsson, S.A., Sigurdsson, A., Jonasdottir, A., Jonasdottir, A., et al. (2012). Rate of de novo mutations and the importance of father's age to disease risk. Nature 488, 471-475.

Li, H., and Durbin, R. (2011). Inference of human population history from individual whole-genome sequences. Nature 475, 493-496.

Li, H., and Stephan, W. (2006). Inferring the demographic history and rate of adaptive substitution in Drosophila. PLoS Genet. 2, e166.

Li, H., Xiang-Yu, J., Dai, G., Gu, Z., Ming, C., Yang, Z., Ryder, O.A., Li, W.H., Fu, Y.X., and Zhang, Y.P. (2016). Large numbers of vertebrates began rapid population decline in the late 19th century. Proc. Natl. Acad. Sci. USA 113, 14079-14084. 
Lisiecki, L.E., and Raymo, M.E. (2005). A Pliocene-Pleistocene stack of 57 globally distributed benthic $\delta 180$ records. Paleoceanography 20, PA1003.

Liu, X., and Fu, Y.X. (2020). Stairway Plot 2: demographic history inference with folded SNP frequency spectra. Genome Biol. 21, 280.

Liu, X.M., and Fu, Y.X. (2015). Exploring population size changes using SNP frequency spectra. Nat. Genet. 47, 555-559.

Lopez, M., Kousathanas, A., Quach, H., Harmant, C., Mouguiama-Daouda, P., Hombert, J.M., Froment, A., Perry, G.H., Barreiro, L.B., Verdu, P., et al. (2018). The demographic history and mutational load of African hunter-gatherers and farmers. Nat. Ecol. Evol. 2, 721-730.

Manica, A., Amos, W., Balloux, F., and Hanihara, T. (2007). The effect of ancient population bottlenecks on human phenotypic variation. Nature 448, 346-348.

Melchionna, M., Profico, A., Castiglione, S., Sansalone, G., Serio, C., Mondanaro, A., Di Febbraro, M., Rook, L., Pandolfi, L., and Di Vincenzo, F. (2020). From smart apes to human brain boxes. A uniquely derived brain shape in late hominins clade. Front. Earth Sci. 8, 273.

Myers, S., Fefferman, C., and Patterson, N. (2008). Can one learn history from the allelic spectrum? Theor. Popul. Biol. 73, 342-348.

Ni, X.J., Ji, Q., Wu, W.S., Shao, Q.F., Ji, Y.N., Zhang, C., Liang, L., Ge, J.Y., Guo, Z., Li, J.H., et al. (2021). Massive cranium from Harbin in northeastern China establishes a new Middle Pleistocene human lineage. The Innovation 100130.

Nielsen, R., Akey, J.M., Jakobsson, M., Pritchard, J.K., Tishkoff, S., and Willerslev, E. (2017). Tracing the peopling of the world through genomics. Nature 541, 302-310.

Pedersen, M.E.H. (2010). Tuning and simplifying heuristical optimization. PhD thesis, Univ. Southampton.

Polanski, A., Bobrowski, A., and Kimmel, M. (2003). A note on distributions of times to coalescence, under time-dependent population size. Theor. Popul. Biol. 63, 33-40.

Profico, A., Di Vincenzo, F., Gagliardi, L., Piperno, M., and Manzi, G. (2016). Filling the gap. Human cranial remains from Gombore II (Melka Kunture, Ethiopia; ca. $850 \mathrm{ka}$ ) and the origin of Homo heidelbergensis. J. Anthropol. Sci. 94, 41-63.

Prugnolle, F., Manica, A., and Balloux, F. (2005). Geography predicts neutral genetic diversity of human populations. Curr. Biol. 15, R159-160.

Ramachandran, S., Deshpande, O., Roseman, C.C., Rosenberg, N.A., Feldman, M.W., and Cavalli-Sforza, L.L. (2005). Support from the relationship of genetic and geographic distance in human populations for a serial founder effect originating in Africa. Proc. Natl. Acad. Sci. USA 102, 15942-15947.

Reich, D., Green, R.E., Kircher, M., Krause, J., Patterson, N., Durand, E.Y., Viola, B., Briggs, A.W., Stenzel, U., Johnson, P.L.F., et al. (2010). Genetic history of an archaic hominin group from Denisova Cave in Siberia. Nature 468, 1053-1060.

Scally, A., and Durbin, R. (2012). Revising the human mutation rate: implications for understanding human evolution. Nat. Rev. Genet. 13, 745-753.

Schiffels, S., and Durbin, R. (2014). Inferring human population size and separation 

history from multiple genome sequences. Nat. Genet. 46, 919-925.

Schlebusch, C.M., and Jakobsson, M. (2018). Tales of human migration, admixture, and selection in Africa. Annu. Rev. Genomics Hum. Genet. 19, 405-428.

Shao, Q.F., Ge, J.Y., Ji, Q., Li, J.H., Wu, W.S., Ji, Y.N., Zhan, T., Zhang, C., Li, Q., Grün, R., et al. (2021). Geochemical provenancing and direct dating of the Harbin archaic human cranium. The Innovation 100131.

Skoglund, P., Thompson, J.C., Prendergast, M.E., Mittnik, A., Sirak, K., Hajdinjak, M., Salie, T., Rohland, N., Mallick, S., Peltzer, A., et al. (2017). Reconstructing prehistoric African population structure. Cell 171, 59-71.

Stoneking, M., and Krause, J. (2011). Learning about human population history from ancient and modern genomes. Nat. Rev. Genet. 12, 603-614.

Stringer, C. (2016). The origin and evolution of Homo sapiens. Philos. Trans. R. Soc. Lond., Ser. B: Biol. Sci. 371, 20150237.

Tajima, F. (1989). Statistical method for testing the neutral mutation hypothesis by DNA polymorphism. Genetics 123, 585-595.

Terhorst, J., Kamm, J.A., and Song, Y.S. (2017). Robust and scalable inference of population history from hundreds of unphased whole genomes. Nat. Genet. 49, 303-309.

White, T.D., Asfaw, B., DeGusta, D., Gilbert, H., Richards, G.D., Suwa, G., and Howell, F.C. (2003). Pleistocene Homo sapiens from Middle Awash, Ethiopia. Nature 423, 742-747.

Yu, D., Dong, L., Yan, F., Mu, H., Tang, B., Yang, X., Zeng, T., Zhou, Q., Gao, F., Wang, Z., et al. (2019). eGPS 1.0: comprehensive software for multi-omic and evolutionary analyses. Natl. Sci. Rev. 6, 867-869.

Zivković, D., and Wiehe, T. (2008). Second-order moments of segregating sites under variable population size. Genetics 180, 341-357. 
STAR $\star$ METHODS

499

KEY RESOURCES TABLE

\begin{tabular}{|c|c|c|}
\hline REAGENT or RESOURCE & SOURCE & IDENTIFIER \\
\hline \multicolumn{3}{|c|}{ Deposited data } \\
\hline \multirow[t]{2}{*}{1000 Genomes project data, phase 3} & 1000 Genomes & http://ftp.1000genomes.ebi.ac.uk/vol \\
\hline & project & 1/ftp/release/20130502/ \\
\hline Human Genome Diversity Project - & HGDP and CEPH & ftp://ngs.sanger.ac.uk/production/hg \\
\hline Centre d'Etude du Polymorphisme & & $\mathrm{dp}$ \\
\hline \multicolumn{3}{|l|}{ Humain (HGDP-CEPH) panel } \\
\hline Observed SFSs and raw data to prepare & This study & https://data.mendeley.com/datasets/x \\
\hline \multirow[t]{2}{*}{ Figures } & & mf5r8nzrn/draft?a=8e2a5abe-de47-4 \\
\hline & & ab7-a313-e2e5526cbc55 \\
\hline \multicolumn{3}{|c|}{ Software and algorithms } \\
\hline \multirow[t]{4}{*}{ FitCoal } & This study & https://www.picb.ac.cn/evolgen/, \\
\hline & & https://zenodo.org/record/4805461\#. \\
\hline & & YN161Ey-vuo and \\
\hline & & http://www.egps-software.net/ \\
\hline
\end{tabular}

\section{CONTACT FOR REAGENT AND RESOURCE SHARING}

503 Further information and requests for resource and reagents should be directed to and will be fulfilled by the Lead Contact, Haipeng Li (lihaipeng@ picb.ac.cn).

\section{METHOD DETAILS}

\section{Standard coalescent time and time in generations}

The population size is denoted $N(\cdot)$, representing the demographic history.

509 Time $\tau$ represents one-point scaled time since the time in a generation is scaled by

$5102 N(0)$. Time $t$ is usually scaled by $2 N(t)$ generations (Bhaskar and Song, 2014;

511 Chen, 2019; Fu, 1995; Myers et al., 2008). To distinguish it from the one-point scaled

512 time $\tau$, time $t$ is designated as the standard coalescent time. 


\section{Fast infinitesimal time coalescent (FitCoal) process}

The FitCoal calculates the expected branch length for each type of site frequency spectrum (SFS) under arbitrary demographic history $N(\cdot)$. We assume that a sample is obtained by randomly taken $n$ sequences from the population. The sample is designated to be state $l(l=2, \cdots, n)$ at time $t$ if it has exactly $l$ ancestral lineages at this time. The probability of state $l$ at time $t$ is denoted $p_{l}(t)$. In a coalescent tree, a branch is designated to be type $i$ if it has exactly $i$ descendants. We have

$$
\frac{\mathrm{d}}{\mathrm{d} t} p_{l}(t)=\left\{\begin{array}{ll}
\left(\begin{array}{c}
l+1 \\
2
\end{array}\right) p_{l+1}(t)-\left(\begin{array}{c}
l \\
2
\end{array}\right) p_{l}(t) & l=2, \cdots, n-1 \\
-\left(\begin{array}{c}
l \\
2
\end{array}\right) p_{l}(t) & l=n
\end{array} .\right.
$$

When $\Delta t$ is extremely small (Figure 1), there is at most one coalescent event during $t$ and $t+\Delta t$, leading to

$$
p_{l}(t+\Delta t)=\left\{\begin{array}{ll}
\left(\begin{array}{c}
l+1 \\
2
\end{array}\right) \Delta t p_{l+1}(t)+\left(1-\left(\begin{array}{c}
l \\
2
\end{array}\right) \Delta t\right) p_{l}(t) & l=2, \cdots, n-1 \\
\left(1-\left(\begin{array}{l}
l \\
2
\end{array}\right) \Delta t\right) p_{l}(t) & l=n
\end{array} .\right.
$$

The branch length is in units of generations. The expected branch length of state $l$ during $t$ and $t+\Delta t$ is calculated as $\int_{t}^{t+\Delta t} 2 N(t) p_{l}(t) l \mathrm{~d} t$. The probability that a branch of state $l$ is of type $i$ is $\frac{\left(\begin{array}{c}n-i-1 \\ l-2\end{array}\right)}{\left(\begin{array}{c}n-1 \\ l-1\end{array}\right)}(\mathrm{Fu}, 1995)$. The expected branch length of type $i$ of state $l$ during $t$ and $t+\Delta t$ is $\int_{t}^{t+\Delta t} 2 N(t) p_{l}(t) l \frac{\left(\begin{array}{c}n-i-1 \\ l-2\end{array}\right)}{\left(\begin{array}{c}n-1 \\ l-1\end{array}\right)} \mathrm{d} t$. Therefore, the expected branch length $B L_{i}(N(\cdot))$ of type $i$ is

$$
\sum_{l=2}^{n-i+1} \int_{0}^{\infty} 2 N(t) p_{l}(t) l \mathrm{~d} t \frac{\left(\begin{array}{c}
n-i-1 \\
l-2
\end{array}\right)}{\left(\begin{array}{c}
n-1 \\
l-1
\end{array}\right)} .
$$

A FitCoal time partition is denoted by $\left\{t_{0}, t_{1}, \cdots, t_{m}\right\}$, where $0=t_{0}<t_{1}<$ $\cdots<t_{m}$. We have $p_{l}\left(t_{0}\right)=\left\{\begin{array}{ll}1 & l=n \\ 0 & \text { else }\end{array}\right.$. For a large positive number $m$, if $t_{m}$ is large and $\left(t_{k}-t_{k-1}\right)$ is small for $k=1, \cdots, m$, then

$$
p_{l}\left(t_{k}\right)= \begin{cases}\left(1-\left(\begin{array}{l}
l \\
2
\end{array}\right)\left(t_{k}-t_{k-1}\right)\right) p_{l}\left(t_{k-1}\right) & l=n \\
\left(1-\left(\begin{array}{l}
l \\
2
\end{array}\right)\left(t_{k}-t_{k-1}\right)\right) p_{l}\left(t_{k-1}\right)+\left(\begin{array}{c}
l+1 \\
2
\end{array}\right)\left(t_{k}-t_{k-1}\right) p_{l+1}\left(t_{k-1}\right) & \text { else }\end{cases}
$$

where $k=1, \cdots, m$.

The expected branch length of type $i$ is calculated as

$$
B L_{i}(N(\cdot))=\sum_{l=2}^{n-i+1} l \frac{\left(\begin{array}{c}
n-i-1 \\
l-2
\end{array}\right)}{\left(\begin{array}{c}
n-1 \\
l-1
\end{array}\right)}\left(\sum_{k=1}^{m} 2 N\left(t_{k-1}\right) p_{l}\left(t_{k-1}\right)\left(t_{k}-t_{k-1}\right)\right) .
$$

To determine the time partition, we required that the coalescent probability was less than $10^{-4}$ during $t_{k-1}$ and $t_{k}(k=1, \cdots, m)$, the probability of common 
ancestor (i.e., the probability of state 1$)$ at $t_{m}$ was larger than $\left(1-10^{-6}\right)$. When the sample size was 10 , the number of infinitesimal time intervals was $1,571,200$. When the sample size was 200, the number of infinitesimal time intervals was 7,038,398. Thus, each $\Delta t$ was extremely small for precise calculation of expected branch length, and the time was partitioned to obtain $p_{l}(t)$ in order to calculate the expected branch length of type $i$.

\section{Tabulated FitCoal}

The expected branch length of each type can be calculated for arbitrary time intervals according to the procedure described above. Considering another tabulated time partition $\left\{t_{0}, t_{1}, \cdots, t_{m}\right\}\left(0=t_{0}<t_{1}<\cdots<t_{m}\right)$, the expected branch length of a type is equal to the sum of the expected branch length of this type during each tabulated time interval, thus the latter can be rescaled and tabulated.

The scaled expected branch length $B L_{i, t}$ of type $i$ during 0 and $t$ is $B L_{i, t}=\sum_{l=2}^{n-i+1} \int_{0}^{t} p_{s}(l) l \frac{\left(\begin{array}{c}n-i-1 \\ l-2\end{array}\right)}{\left(\begin{array}{c}n-1 \\ l-1\end{array}\right)} \mathrm{d} s$, where $i=1, \cdots, n-1$. For the tabulated time partition $\left\{t_{0}, t_{1}, \cdots, t_{m}\right\}, B L_{i, t_{0}}, B L_{i, t_{1}}, \cdots$, and $B L_{i, t_{m}}$ are tabulated. When $n=10$, $m=231$. When $n=200, m=529$.

$B L_{i, t}$ is used to calculate the expected branch lengths under arbitrary demographic histories. When $\tilde{t} \in\left[t_{k-1}, t_{k}\right)$,

$$
B L_{i, \tilde{t}} \approx \frac{t_{k}-\tilde{t}}{t_{k}-t_{k-1}} B L_{i, t_{k-1}}+\frac{\tilde{t}-t_{k-1}}{t_{k}-t_{k-1}} B L_{i, t_{k}} .
$$

If $N(t)$ is a piecewise constant, that is, there exists a demographic time partition $\left\{\tilde{t}_{0}, \tilde{t}_{1}, \cdots, \tilde{t}_{\tilde{m}}\right\}$, such that $N(t)=N_{k}$ for $t \in\left[\tilde{t}_{k}, \tilde{t}_{k+1}\right), k=0, \cdots, \widetilde{m}$. Then, the expected branch length of type $i$ is calculated as

$$
B L_{i}(N(\cdot))=\sum_{k=1}^{\widetilde{m}} 2 N_{k}\left(B L_{i, \tilde{t}_{k}}-B L_{i, \tilde{t}_{k-1}}\right) \text {. }
$$

When $N(t)$ is complex, the population size can be approximated by a piecewise constant function.

\section{Composite likelihood}

The mutation rate per base pair per generation is denoted $\mu$, and $\vec{\xi}=\left(\xi_{i}\right)$ is the observed number of SNPs of $n$ sequences with $\sigma$ base pairs, where $i=1, \cdots, n-1$. The expected SFS is $\vec{\lambda}=\left(\lambda_{i}\right)$, where $\lambda_{i}=\mu \sigma B L_{i}(N(\cdot))$. Following the Poisson 
probability and previous studies ( $\mathrm{Li}$ and Stephan, 2006), the composite likelihood is calculated as follows:

$$
L_{\mu, \iota}(\vec{\xi}, N(\cdot))=\prod_{i=1}^{n-1} \frac{\lambda_{i}^{\xi_{i}} e^{-\lambda_{i}}}{\xi_{i} !} .
$$

The likelihood is extended to missing data and truncated SFS (see Supplemental Text).

\section{Demographic inference}

The number of demographic time intervals is variable. FitCoal first fits the observed SFS using a constant size model with one demographic time interval, and the number of time intervals is increased by one at a time to generate more complex models. The Local Unimodal Sampling (LUS) algorithm (Pedersen, 2010) is used to maximize the likelihood and estimate demographic parameters. A log-likelihood promotion rate is used to determine the best model to explain the observed SFS, and $20 \%$ is used as the threshold.

A series of demography with $m$ pieces is denoted by a set $S(m)$, where $S(m)$ contains all of the following $m$ pieces of population size:

$$
\begin{aligned}
& N\left(t \mid N 0>0, N_{(m)}, t_{(m)}, c_{(m)}\right) \\
& =\left\{\begin{array}{cl}
N_{m} N 0 & t \geq t_{m} \\
N_{k} N 0 & t_{k} \leq t<t_{k+1}, c_{k} \in \mathcal{C}, k=1, \cdots, m-1, \\
\frac{\left(t_{k+1}-t_{k}\right) N_{k+1} N_{k} N 0}{\left(t-t_{k}\right) N_{k}+\left(t_{k+1}-t\right) N_{k+1}} & t_{k} \leq t<t_{k+1}, c_{k} \in \mathcal{E}, k=1, \cdots, m-1
\end{array}\right.
\end{aligned}
$$

where $N_{(m)}=\left(N_{1}, \cdots, N_{m}\right) \in N[m], t_{(m)}=\left(t_{1}, \cdots, t_{m}\right) \in t[m]$,

$c_{(m)}=\left(c_{1}, \cdots, c_{m}\right) \in c[m], N[m]=\left\{\left(N_{1}, \cdots, N_{m}\right) \mid N_{1}=1, N_{i}>0\right.$ for $\left.i>1\right\}$,

$t[m]=\left\{\left(t_{1}, \cdots, t_{m}\right) \mid 0=t_{1}<\cdots<t_{m}\right\}, c[m]=\left\{\left(c_{1}, \cdots, c_{m}\right) \mid c_{m} \in \mathcal{C}, c_{i} \in \mathcal{C} \cup\right.$

$\mathcal{E}$ for $=1, \cdots, m-1\}, \mathcal{C}=\{$ constant $\}$, and $\mathcal{E}=\{$ exponential $\}$.

The set $S(m)$ was used as the wide-range parameter space to determine the maximum likelihood. To find the best demographic history to explain the observed SFS, the following procedures were used:

(1) The number of inference time intervals (or pieces) $m$ is initially set to 1 , and the maximum likelihood max $L_{1}$ is determined with the constant size model (model in $S(1))$.

(2) Increase $m$ by 1 . For each change of type $c_{(m)}$, parameters $N_{(m)}=\left(N_{1}, \cdots, N_{m}\right)$ and $t_{(m)}=\left(t_{1}=0, t_{2}, \cdots, t_{m}\right)$ are searched to maximize the likelihood by LUS 
600

601

602

603

604

605

606

607

608

609

610

611

612

613

614

615

616

617

618

619

620

621

622

623

624

625

626

627

628

629

630

631

algorithm to fit the observed SFS. The maximum likelihood $\max L_{m}$ is calculated with models in $\mathrm{S}(\mathrm{m})$ with all possible change types.

(3) Repeat step (2) until $(1+$ threshold $) \cdot \log \left(\max L_{m}\right)<\log \left(\max L_{m-1}\right)$ is obtained. The best model corresponding $\max L_{m-1}$ is determined to explain the observed SFS.

(4) To avoid local optima, steps (1) - (3) are repeated $K$ times to find the best model. $K=10$ when analyzing simulated samples, and $K=200$ when analyzing the observed SFSs of the 1000GP and HGDP-CEPH populations.

To determine the threshold of log-likelihood promotion rate, a large number of simulations were performed (Table S9). For each model, 200 replicates were conducted, and the number of inference time intervals in the estimated demographic history was determined for each replicate. If the estimated number of inference time intervals was larger than the true number of inference time intervals, overfitting was recorded. When the former was smaller than the latter, underfitting was considered. The thresholds of $10 \%, 20 \%$, and $30 \%$ were used. When $10 \%$ was used, the maximum overfitting rate was $2 \%$. When $20 \%$ was used, all cases examined were inferred correctly. When $30 \%$ was used, the underfitting was observed in one of 20 examined models. Therefore, $20 \%$ was used as the threshold of log-likelihood promotion rate in subsequent analyses.

\section{Data simulation}

Data were simulated using ms (Hudson, 2002) and MaCS (Chen et al., 2009) software. Unless otherwise specified, a generation time was assumed to be 24 years (Liu and Fu, 2015; Scally and Durbin, 2012), the mutation rate $\mu$ was set for $1.2 \times 10^{-8}$ per base per generation (Campbell et al., 2012; Conrad et al., 2011; Kong et al., 2012; Liu and Fu, 2015), and the recombination rate was $r=0.8 \mu$. For each model, 200 SFSs were simulated to calculate the median and 2.5 and 97.5 percentiles. When verifying the inferred demographic histories, 80,000 DNA fragments with the length of $10 \mathrm{~kb}$ each were used for simulation, taking into the consideration of small fragments split by sequencing mask in 1000GP and HGDP-CEPH data sets. High frequency alleles of SFS (10\% mutation types for Bottleneck I, II, III, VII, VIII, IX, and $15 \%$ for Bottleneck IV, V, VI) were removed when assessing models to verify the 
632

633

634

635

636

637

638

639

640

641

642

643

644

645

646

647

648

649

650

651

652

653

654

655

656

657

658

659

660

661

662

663

664

super bottleneck. Detailed simulation command lines and demographic inference are presented in the Supplementary Text.

\section{Genomes Project data}

Sequences of autosomal SNPs in 1000GP phase 3 (Altshuler et al., 2015) were downloaded from the 1000GP ftp server

(ftp://ftp.1000genomes.ebi.ac.uk/vol1/ftp/release/20130502/), and 26 populations were analyzed, including seven African populations (ACB, ASW, ESN, GWD, LWK, MSL, and YRI), five European populations (CEU, FIN, GBR, IBS, and TSI), five East Asian populations (CDX, CHB, CHS, JPT, and KHV), five South Asian populations (BEB, GIH, ITU, PJL, and STU), and four American populations (CLM, MXL, PEL, and PUR). The 1000 GP strict mask was used to exclude artifacts of SNP calling. Noncoding regions except pseudogenes, defined by GENCODE release 35 (Frankish et al., 2019), were examined to avoid potential effects of purifying selection. The number of sites that passed the filtering was $826,649,529$ in the human genome.

Bi-allelic polymorphic sites with high-confidence ancestral allele inference, according to 1000GP annotations, were used. To avoid the effect of positive selection, high frequency mutations were excluded, and the truncated SFS was used to infer demographic history (Figure S11; Table S10). The average proportion of excluded high-frequency SNPs for all 1000GP populations was $4.40 \%$.

\section{HGDP-CEPH data}

In total, 24 populations were analyzed, including three African populations (Biaka, Mandeka, and Yoruba), five European populations (Adygei, Basque, French, Russian, and Sardinain), four Middle East populations (Bedouin, Druze, Mozabite, and Palestinian), three East Asian populations (Han, Japanese, and Yakut), eight Central and South Asian populations (Balochi, Brahui, Burusho, Hazara, Kalash, Makrani, Pathan, and Sindhi), and an American population (Maya). Only bi-allelic SNPs locating in GENCODE non-coding regions (Frankish et al., 2019) except pseudogenes that passed HGDP-CEPH filtering were used. HGDP-CEPH accessible mask was also used to filter SNPs (Bergstrom et al., 2020). The number of sites that passed the filtering was 791,999,125 in the human genome. Missing data were allowed to avoid artifacts due to imputation. The proportion of sites with two or more 
665

666

667

668

669

670

671

672

673

674

675

676

677

678

679

680

681

682

683

684

685

686

687

688

689

690

691

692

693

missing individuals was less than 3\% for all populations (Table S11). Each population had two SFSs, with one calculated from sites with no missing data, and another from sites with one missing individual. Similarly, truncated SFSs were used to avoid the effect of positive selection (Figures S12 and S13; Table S12). The average proportion of excluded high-frequency SNPs for all HGDP-CEPH populations was 7.18\%.

\section{SFS truncation}

Denote the SFS of $n$ samples by $\vec{\lambda}=\left(\lambda_{1}, \ldots, \lambda_{n-1}\right)$. An $m$-dimension vector $\vec{V}=\left(v_{1}, \ldots, v_{m}\right)$ is said to be tail-up if there exist $z \in\{1, \cdots, m-1\}$ such that $v_{z}<\cdots<v_{m}$. If $\vec{\lambda}$ is the expected SFS of a single varying size population, we have $\lambda_{[n / 2]}>\cdots>\lambda_{n-1}$. However, the observed SFS $\vec{\xi}=\left(\xi_{1}, \ldots, \xi_{n-1}\right)$ may be tailed up because of some evolutionary factors, such as positive selection and population structure, which could introduce bias to the demographic inference. Therefore, the truncated SFS is recommended.

A simple procedure is implemented to discard the tail-up types of SFS, containing high-frequency mutations. To determine the truncated tail of SFS, a small window slides through the SFS. The cutoff is determined if $\xi_{i}$ exceeds its random fluctuation range. Let $\widehat{n}(\vec{\xi})=\max _{k \in\{1, \cdots, n-1\}}\left\{k \mid \bar{w}_{k}(\vec{\xi})-3 S D_{k}(\vec{\xi})<w_{k-i(n)+1}, \cdots, w_{k}<\right.$ $\left.\bar{w}_{k}(\vec{\xi})+3 S D_{k}(\vec{\xi})\right\}$

where $\bar{w}_{k}(\vec{\xi})=\frac{1}{i(n)} \sum_{a=k-i(n)+1}^{k} \xi_{a}, S D_{k}(\vec{\xi})=\sqrt{\bar{w}_{k}(\vec{\xi})}$, and

$i(n)= \begin{cases}3 & n \leq 50 \\ 4 & 50<n \leq 100 . \text { The truncated SFS } \vec{\xi}^{T}=\left(\xi_{i}\right), \text { where } i=1, \cdots, k . \text { In the } \\ 5 & n>100\end{cases}$ analysis, we used this strategy to truncate the SFS for each human population. We call $(n-k) / n$ the proportion of truncated SFS types.

When the truncating strategy was applied, the proportion of truncated SFS types was different for different populations (Table S5, S7). Therefore, to verify the effect of this strategy, the same truncating standard ( $10 \%$, the mean proportion) was also used for 1000GP populations (Figure S15). For HGDP-CEPH, because the proportion of considered SNPs without missing samples is larger than $80 \%$ for all populations, we used the corresponding SFS to determine the cutoff to truncate both SFSs. 
694 Similarly, the same truncating standard ( $15 \%$, the mean proportion) was used for

695

696

697

698

699

700

701

702

703

704

705

706

707

708

709 HGDP-CEPH (Figure S15).

\section{Composite likelihood}

Denote $\mu$ as the mutation rate per base pair per generation. Denote $\vec{\xi}=\left(\xi_{i}\right)$ as the observed number of SNPs of $n$ sequences with $\sigma$ base pair, where $i=$ $1, \cdots, n-1$. The expected SFS $\vec{\lambda}=\left(\lambda_{i}\right)$, where $\lambda_{i}=\mu \sigma B L_{i}(N(\cdot))$. Following the Poisson probability and the previous studies (Hudson, 2001; Li and Stephan, 2006), the composite likelihood could be written as

$$
L_{\mu, \iota}(\vec{\xi}, N(\cdot))=\prod_{i=1}^{n-1} \frac{\lambda_{i}^{\xi_{i}} e^{-\lambda_{i}}}{\xi_{i} !} .
$$

For missing data, we assume that $\sigma^{(n)}$ base pair are sequenced in $n$ samples and $S$ is the set of all sample sizes. We denote the observed number of SNPs of $n(\in S)$ sequences by $\vec{\xi}^{(n)}=\left(\xi_{1}^{(n)}, \cdots, \xi_{n-1}^{(n)}\right)$. The expected SFS of $n$ sequences $\vec{\lambda}^{(n)}=\left(\lambda_{1}^{(n)}, \cdots, \lambda_{n-1}^{(n)}\right)$, where $\lambda_{i}^{(n)}=\mu \sigma^{(n)} B L_{i}^{(n)}(N(\cdot)), B L_{i}^{(n)}(N(\cdot))$ is the expected branch length of type $i$ with $n$ samples under population size $N(\cdot)$. Total number of base pair is given by $\sigma(S):=\sum_{n \in S} \sigma^{(n)}$. The composite likelihood could be written as

$$
\begin{aligned}
& \left.L_{\mu,(\iota(\ell)}\right)_{n \in S}\left(\left(\vec{\xi}^{(n)}\right)_{n \in S}, N(\cdot)\right) \\
& =\prod_{n \in S} L_{\mu, \iota}(n)\left(\vec{\xi}^{(n)}, N(\cdot)\right) \\
& =\prod_{n \in S} \prod_{i=1}^{n-1} \frac{\left(\lambda_{i}^{(n)}\right)^{\xi_{i}^{(n)}} e^{-\lambda_{i}^{(n)}}}{\xi_{i}^{(n)} !}
\end{aligned}
$$

If SFS is tail-up, we use truncated SFS $\vec{\xi}^{T}=\left(\xi_{i}\right)$, where $i=1, \cdots, k$. The composite likelihood is

$$
L_{\mu, \iota}\left(\vec{\xi}^{T}, N(\cdot)\right)=\prod_{i=1}^{k} \frac{\lambda_{i}^{\xi_{i}} e^{-\lambda_{i}}}{\xi_{i} !} .
$$

Sequencing errors often affect rare mutations in a sample. Thus singletons and mutations with size $(n-1)$ can be discarded. Although this is unnecessary in this 
study, as a general method, the composite likelihood of an SFS without those mutations is

$$
L_{\mu, \iota}(\vec{\xi}, N(\cdot))=\prod_{i=2}^{n-2} \frac{\lambda_{i}^{\xi_{i}} e^{-\lambda_{i}}}{\xi_{i} !}
$$

\section{Loss of genetic diversity due to the super bottleneck}

To measure the loss of current human genetic diversity due to the super bottleneck, we calculated the expected tree length of demographic histories with or without the super bottleneck. It was straightforward to ignore a bottleneck with instantaneous size changes, thus we considered seven 1000GP African populations (ACB, ASW, ESN, GWD, LWK, MSL and YRI) and one HGDP-CEPH African population (Yoruba). To remove the bottleneck, we replaced the population size during the super bottleneck with that after the bottleneck. We then compared the expected tree length of inferred demographic history $\left(\omega_{1}\right)$ with that of demographic history without the bottleneck $\left(\omega_{0}\right)$.

The loss of current genetic diversity due to the super bottleneck is $\left(\omega_{0}-\right.$ $\left.\omega_{1}\right) / \omega_{0}$. When the actual sample size was used for each population, the genetic diversity was measured as Watterson's $\theta$. The genetic diversity loss of these eight populations was $46.22 \%$ and the range was $32.17-60.56 \%$.

When $n=2$, the genetic diversity was measured as $\pi$, the pairwise nucleotide diversity. The loss of current genetic diversity in these eight populations was $65.85 \%$ and the range was $52.71-73.60 \%$. It was larger than the estimate based on Watterson's $\theta$ because the bottleneck was ancient and the recovery rate of Watterson's $\theta$ was faster than that of $\pi$ (Tajima, 1989). These results demonstrate the importance of the super bottleneck in the human evolution.

\section{QUANTIFICATION AND STATISTICAL ANALYSES}

\section{Validation of FitCoal calculation}

We verified the calculation of expected branch lengths in this section. Under the constant size model, when the sample size was small ( $n=5$, where $n$ is the number 
of sequences) or extremely large $(n=1,000)$, FitCoal calculated the expected branch lengths correctly (Fu, 1995) (Figure S14, Table S13). Computational accuracy reaches $10^{-8}$ or $10^{-11}$. The high accuracy is important for the precise estimation of demographic history in the following sections.

Moreover, our results were almost the same as the expected branch lengths under three simple models calculated by using the Zivković-Wiehe method (Zivković and Wiehe, 2008) (Table S14). Since Zivković-Wiehe equations can be numerically solved when $\mathrm{n}<50$, we could not compare our results with theirs when the sample size was large.

For more complex models, the average branch lengths were obtained from extensive coalescent simulations. Although with certain variances, the simulated results were consistent with the FitCoal expected branch lengths under different demographic models (Table S15). Therefore, FitCoal can analytically derive the expected branch length for each SFS type under arbitrary demographic models.

We also compared the results obtained from the tabulated FitCoal and those from the original ones without tabulation. These results were nearly identical with each other (Tables S14 and S15). Since the former was much faster than the latter, the former was used to infer demographic histories. Hereafter, tabulated FitCoal is referred to as FitCoal for short, unless otherwise indicated.

\section{FitCoal- and simulation-based likelihood surface}

In this section, we compared two likelihood surfaces based FitCoal and simulation (Figure S1). We considered an instantaneous growth model. The population size increases from $10,000\left(N_{1}\right)$ to $20,000\left(N_{0}\right)$ at standard coalescent time 0.2 . For simplicity, we obtained a SFS by multiplying the expected branch length by $\theta l\left(=4 \mathrm{~N}_{0} \mu\right)$, where $\mu l=1.0$. The number of sequences is 100 .

We then compared the FitCoal composite likelihood surface of the SFS and the composite likelihood surface of the SFS based on simulation approach. To draw the likelihood surfaces, we performed a grid search in a parameter space. We considered that the population size increase from $N_{1}$ to $N_{0}$ at standard coalescent time 0.2 , where $N_{0}$ ranges from 19,600 to 20,400 and $N_{1}$ from 9,800 to 10,200 . The 
coalescent simulations were conducted by the ms software. The number of simulations is 100,000 to calculate the simulation-based likelihood.

The surface of FitCoal likelihood is smooth, but the surface of likelihood based on simulation approach is rugged (Figure S1). Moreover, the FitCoal likelihoods are also larger than those based on simulation approach because the FitCoal expected branch lengths fit the data better than the average branch lengths obtained from simulations.

\section{Demographic inference on simulated data}

It has been shown that FitCoal can precisely estimate the demographic histories under six different demographic models (Figure 2). We then validated the accuracy of FitCoal on more simulated data in this section.

Comparing with the examined cases (Figure 2), the performance of FitCoal can be further improved by providing a priori knowledge. In some circumstances, a slow and continuous change may be more biological relevant than a quick and sudden change and vice versa. FitCoal was then re-performed conditional on either exponential or instantaneous change within each inference time interval (Figures S16 and S17). Our results showed that the FitCoal accuracy was enhanced in the presence of correct priori knowledge. Even if the condition was misspecified, the inferred demographic histories were still similar with the true histories.

FitCoal is a model-flexible method and the number of inference time intervals is dependent on the complexity of true demography. FitCoal has the power to detect more complex population histories (Figure S18). Although FitCoal may omit slight changes of population size occurred in short time periods, it has great ability to detect the major changes in all examined complex histories. When two-population split models are considered (Figure S19), FitCoal is reasonably accurate but with a slightly larger recent population size due to the effects of migration.

\section{Effects of positive selection}


To simulate samples affected by positive selection, we considered a two-locus model (Kim and Stephan, 2002) under a constant size model. We assumed that the effective population size was 27,000 , and the number of neutral fragments were 10,000 , and 10 or $20 \%$ of them were partially linked with selected alleles. The distance between the neutral and the selected loci was $50 \mathrm{~kb}$, and recombination rate was $1 \mathrm{cM}$ per $\mathrm{Mb}$. The sample size was 202 (the average sample size of 1000GP populations). The selection coefficient ( $s=0.01$ or 0.05 ) was varied. We assumed a mutation rate of $1.2 \times 10^{-8}$ per base per generation and a generation time of 24 years. To compare among different cases, the fixed number SNPs $(5,882,885$ SNPs, the average number of SNPs in 1000GP populations) were applied. Under neutrality, it was equivalent to the sequenced length of $771.589 \mathrm{Mb}$.

All the simulated samples had a tail-up feature because of the excess of high-frequency mutations (Fay and $\mathrm{Wu}, 2000$ ). Considering the low genetic diversity of selected loci, the contribution of selected loci to the genome-wide diversity was relatively low, thus only a slight excess of rare mutations (Fu and $\mathrm{Li}, 1993)$ was observed. The ratio between the number of singletons and doubletons ranged between 2.01 and 2.10 in the simulated samples, only slightly larger than the expected value (2.0) under neutrality.

We then applied FitCoal to estimated demography. When the full SFSs were used, our results showed that the population size remains constant within 2,000 kry (Figure S5A). If the selection strength was greatly strong ( $s=0.05$, where $s$ is the selection coefficient), FitCoal estimated a large ancient population $\sim 240 \mathrm{kyr}$ ago because of the effects of high-frequency mutations. When the high-frequency mutations were removed (i.e. the truncated SFS), the large ancient population size was reduced (Figure S5B). If $s=0.01$ and $20 \%$ loci were subject to positive selection, a slight population expansion was observed, corresponding to the slight excess of rare mutations due to positive selection. Overall, a correct demographic history was estimated within two million years.

\section{Verification of inferred human demographic histories}


To evaluate the precision of the inferred human demographic histories (Figure 3), we simulated 200 data sets under each demographic history. The SFSs of simulated data fit the observed SFSs perfectly (Figures S20 and S21). The results showed that FitCoal, with truncated SFS, is highly accurate to reveal human demographic history (Figures S22 - S32). Moreover, when high-frequency mutations were discarded, the truncated proportion of SFS was different for different populations. To address the influence of truncated proportions, we inferred the demographic histories by setting the average truncating proportion within each data set (10\% for $1000 \mathrm{GP}$ and $15 \%$ for HGDP-CEPH) (Fig S10). Results were consistent with the ones obtained above. Therefore, the strategy of truncating SFS does not affect our conclusions.

Similar with the log-likelihood ratio test, the number of inference time intervals was determined by the log-likelihood promotion rate when increasing the number of inference time intervals. It is recommended to use $20 \%$ as the threshold of log-likelihood promotion rate derived from extensive simulation results (Table S11). When analyzing the human data, the inferred demographic histories are not sensitive to this threshold (Figure S33, S34; Tables S16, S17). For example, the log-likelihood promotion rate for three and four inference time intervals of CEU is 2471.16 and $17.07 \%$, respectively. The number of inference time intervals is three, and the inferred demographic history is highly similar with that with four inference time intervals. Thus, the inferred demographic histories are robust to the threshold of $20 \%$.

\section{The super bottleneck estimated in Africans}

In this section, we explored why the super bottleneck can only be estimated in the African population and provided the mathematical explanation. We proved that the inferred number of intervals before time $t$ depends on the dimension of the SFS before time $t$.

Denote the probability of state $l$ at time $t$ from $n$ samples by $p_{l}^{n}(t)$, where $l=2, \cdots, n$. And denote the expected brach length of size $i$ from $n$ samples by $B L_{i}^{n}(N(\cdot))$, where $i=1, \cdots, n-1$. There exists an invertible matrix $\mathcal{X}=$ $\left(x_{g}^{h}\right)_{g, h=2, \cdots, n}$ which only depends on $n$, such that $p_{l}^{n}(t)=\sum_{g=2}^{n} x_{g}^{l} p_{g}^{g}(t)$ (Bhaskar 
and Song, 2014; Polanski et al., 2003). If positive numbers $m<n$, there exist a matix $\mathcal{Y}=\left(y_{g}^{h}\right)_{g=2, \cdots, m, h=2, \cdots, n}$, which only depends on $m$ and $n$, such that $p_{l}^{m}(t)=\sum_{h=2}^{n} y_{l}^{h} p_{h}^{n}(t)$. Combined with eq(1), there exist a matrix $Z=\left(z_{g}^{h}\right)_{g=1, \cdots, m-1, h=1, \cdots, n-1}$, which only depends on $m$ and $n$, such that $B L_{i}^{m}(N(\cdot))=\sum_{j=1}^{n-1} z_{i}^{j} B L_{j}^{n}(N(\cdot))$.

Define the population size before time $t$ by $N^{t}(s)=N(t+s)$. Denote the expected branch length of state $l$ before time $t$ by $B_{l}(t)=\left(b_{1, l}(t), \cdots, b_{l-1, l}(t)\right)$, where $b_{i, l}(t)$ represent the expected branch length of state $l$ before time $t$ of type $i$ at time $t$. We have $b_{j, l}(t)=p_{l}^{n}(t) B L_{j}^{l}\left(N^{t}(\cdot)\right) . B L_{i, k}^{t}(i=1, \cdots, n-1)$ denote the branch length of type $i$ whose number of lineages are no more than $k$ before time $t$. We have

$$
\begin{gathered}
B L_{i, k}^{t}=\sum_{l=2}^{k} \sum_{j=1}^{l-1} \frac{p(j \rightarrow i) p(l-j \rightarrow n-i)}{p(l \rightarrow n)} b_{j, l}(t), \\
\text { where } p(a \rightarrow b)= \begin{cases}\left(\begin{array}{l}
b-1 \\
a-1
\end{array}\right) & b \geq a \geq 1 \\
0 & \text { else }\end{cases}
\end{gathered}
$$

Then,

$$
\begin{aligned}
& B L_{i, k}^{t} \\
= & \sum_{l=2}^{k} \sum_{j=1}^{l-1} \frac{p(j \rightarrow i) p(l-j \rightarrow n-i)}{p(l \rightarrow n)} b_{j, l}(t) \\
= & \sum_{l=2}^{k} \sum_{j=1}^{l-1} \frac{p(j \rightarrow i) p(l-j \rightarrow n-i)}{p(l \rightarrow n)} p_{l}^{n}(t) B L_{j}^{l}\left(N^{t}(\cdot)\right) \\
= & \sum_{h=1}^{k-1}\left(\sum_{l=2}^{k} \sum_{j=1}^{l-1} \frac{p(j \rightarrow i) p(l-j \rightarrow n-i)}{p(l \rightarrow n)} p_{l}^{n}(t) z_{j}^{h}\right) B L_{h}^{k}\left(N^{t}(\cdot)\right)
\end{aligned} .
$$

Thus, the space that is generated by $B L_{1, k}^{t}, \cdots, B L_{n-1, k}^{t}$ can be generated by $B L_{1}^{k}\left(N^{t}(\cdot)\right), \cdots, B L_{k-1}^{k}\left(N^{t}(\cdot)\right)$. This leads that the dimension of $\left(B L_{i, k}^{t}\right)_{i=1, \cdots, n-1}$ is no more than $(k-1)$.

If the number of ancestral lineages is no more than $k$ before a given standard coalescent time $t$, the number of inference time intervals should be no more than $(k-1)$ before time $t$ in the inferred demographic history without overfitting. Technically speaking, if a high proportion of the number of ancestral lineages is no more than $k$ before a given standard coalescent time $t$, we have the same conclusion because it is an inferred demographic history.

For the non-African populations, when $t=1.0$, the number of ancestral lineages is no more than three in more than $90 \%$ cases (Table S18), indicating the power to 
892 infer an constant size model (with one inference time interval), an expansion or

893 contraction (with two inference time intervals) beyond this time point. The end time

894 of the super bottleneck is 813 (772-864) kyr ago and the corresponding standard

895 coalescent time is larger than 1.0 for all non-African populations (Figure 3C, F).

896 Therefore, the super bottleneck cannot be inferred in this case since the bottleneck

897 contains three inference time intervals.

898

899

900

901

902

903

904

905

906

907

908

909

910

911

912

913

914

915

916

917

918

919

920

921

\section{Confounding factors of bottleneck}

African populations have complex population structure (Hsieh et al., 2016; Lopez et al., 2018; Schlebusch and Jakobsson, 2018; Skoglund et al., 2017), and a complex population structure model is proposed for African and European populations (Lopez et al., 2018) (Figure S35). To address the effects of population structure, we simulated data for a western rainforest hunter-gatherer (wRHG) and a western farmer (wARG) population and estimated their demographic histories (Figure S35). Due to frequent migrations, a larger recent population size is estimated for both populations. However, the ancient population size $(14,427)$ is accurately inferred for both populations $(14,493$ and 14,428$)$. Thus, the super bottleneck is not due to the complex African population structure.

To consider the effects of archaic introgression from ghost populations (Beerli, 2004; Durvasula and Sankararaman, 2020), we examined different models by assuming that introgression happened in different time periods with different migration rates (Figure S36). Results show that archaic introgression does not result in an ancient super bottleneck.

Truncated SFS was used in demography inference in this study. To examine the effects of SFS truncation, the FitCoal inference was re-performed by taking the full SFSs that include high-frequency derived mutations. Again, the super bottleneck is revealed only in the African populations, but not in the non-African populations (Figures S37 and S38). Therefore, the ancient super bottleneck is not due to the effects of SFS truncation. 


\section{Computational performance}

923 We compared the performance of the FitCoal with or without tabulation. We

924 applied them to analyze the data of YRI population by fixing four inference time

925 intervals and allowing instantaneous population size change. The former is much

926 faster than the latter (1 second vs 36.2 hours).

927

928

DATA AND SOFTWARE AVAILABILITY

929 The authors declare that all data are available in the main text and the

930 supplementary materials. FitCoal is a free plug-in of the eGPS software (Yu et al.,

931 2019) and can be downloaded and run as an independent package. FitCoal and its

932 documentation are available via Zenodo at

933 https://zenodo.org/record/4805461\#.YN161Ey-vuo, our institute website at

934 http://www.picb.ac.cn/evolgen/, and eGPS website http://www.egps-software.net/.

935 Raw data were deposited on Mendeley

936 (https://data.mendeley.com/datasets/xmf5r8nzrn/draft?a=8e2a5abe-de47-4ab7-a313-e

937 2e5526cbc55). 


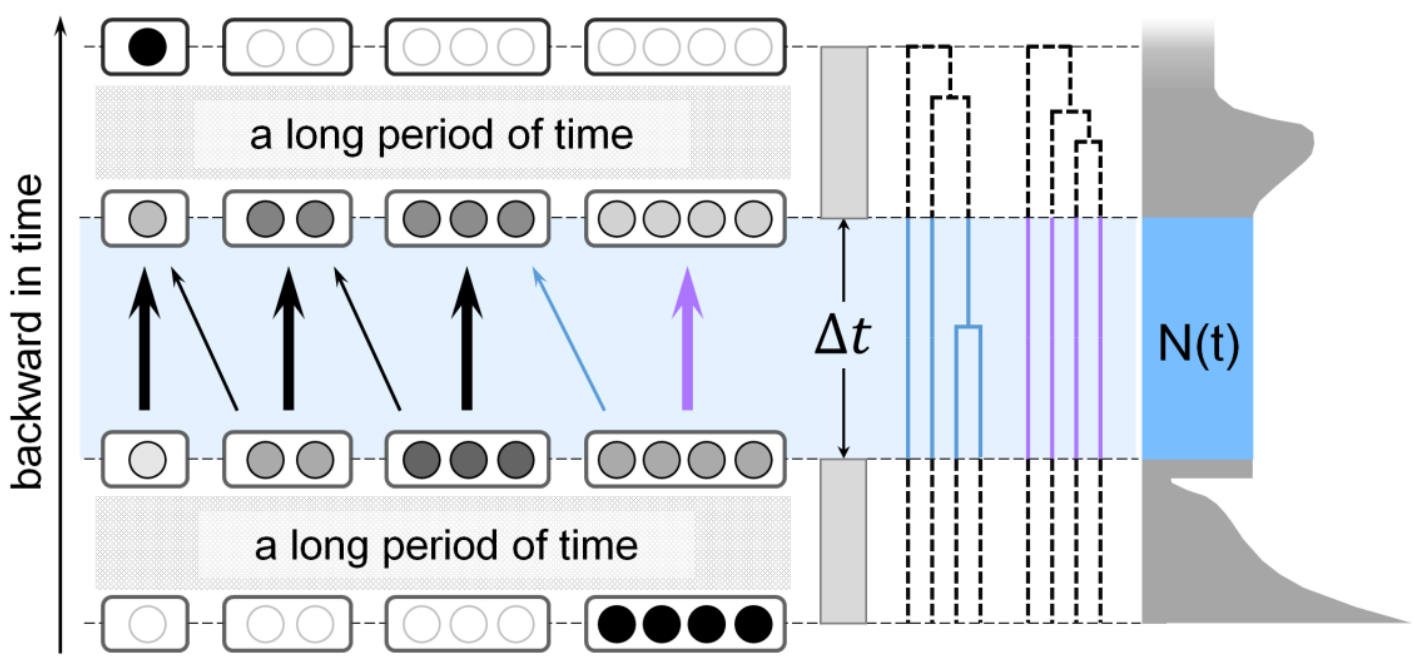

Figure 1. Illustration of the fast infinitesimal time coalescent (FitCoal) process.

941

The left panel shows the backward process in which four lineages coalesce into one after passing through millions of infinitesimal time intervals. The highlighted area shows the backward transformation process of different states with tiny probability changes in an infinitesimal time interval $(\Delta t)$. Thick arrows indicate high transformation probabilities, and thin arrows indicate low transformation probabilities.

Each state is indicated with a rounded rectangle, in which one circle indicates one lineage. The rounded rectangles with black filled circles are the states with probability 1. The rounded rectangles with empty circles are the states with probability 0 . The probabilities between 0 and 1 are indicated by grey circles. The middle panel shows branches of different states. The right panel shows the demographic history of a population. The width of shadowed area indicates the effective population size, i.e., the number of breeding individuals (Harpending et al., 1998). It is assumed that the effective population size remains unchanged within $\Delta t$. 

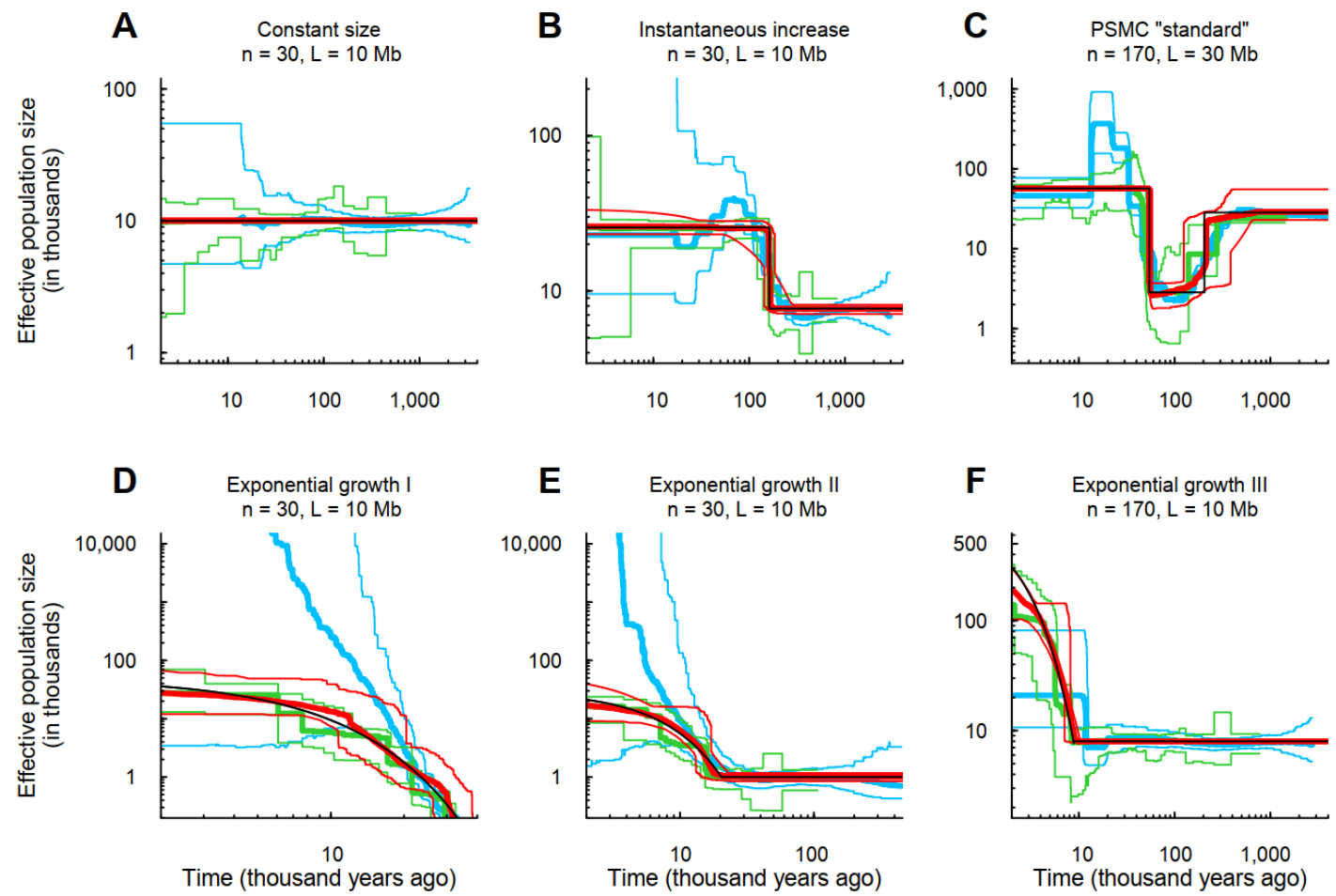

Figure 2. Demographic histories estimated by FitCoal, stairway plot, and PSMC

956

957

958

959

960

961

962

963

964

965

966

using simulated samples. (A) Constant size model. (B) Instantaneous increase model.
(C) PSMC "standard" model.
(D) Exponential growth I model. (E)
(E) Exponential

growth II model. (F) Exponential growth III model. These six models are the same as those of the previous study by Liu and Fu (Liu and Fu, 2015). Thin black lines indicate true models. Thick red lines indicate the medians of FitCoal estimated histories; thin red lines are 2.5 and 97.5 percentiles of FitCoal estimated histories.

Green and blue lines indicate the results of stairway plot and PSMC, respectively, of the previous study (Liu and $\mathrm{Fu}, 2015$ ). The mutation rate is assumed to be $1.2 \times 10^{-8}$ per base per generation, and a generation time is assumed to be 24 years. $n$ is the number of simulated sequences, and $L$ is the length of simulated sequences. 

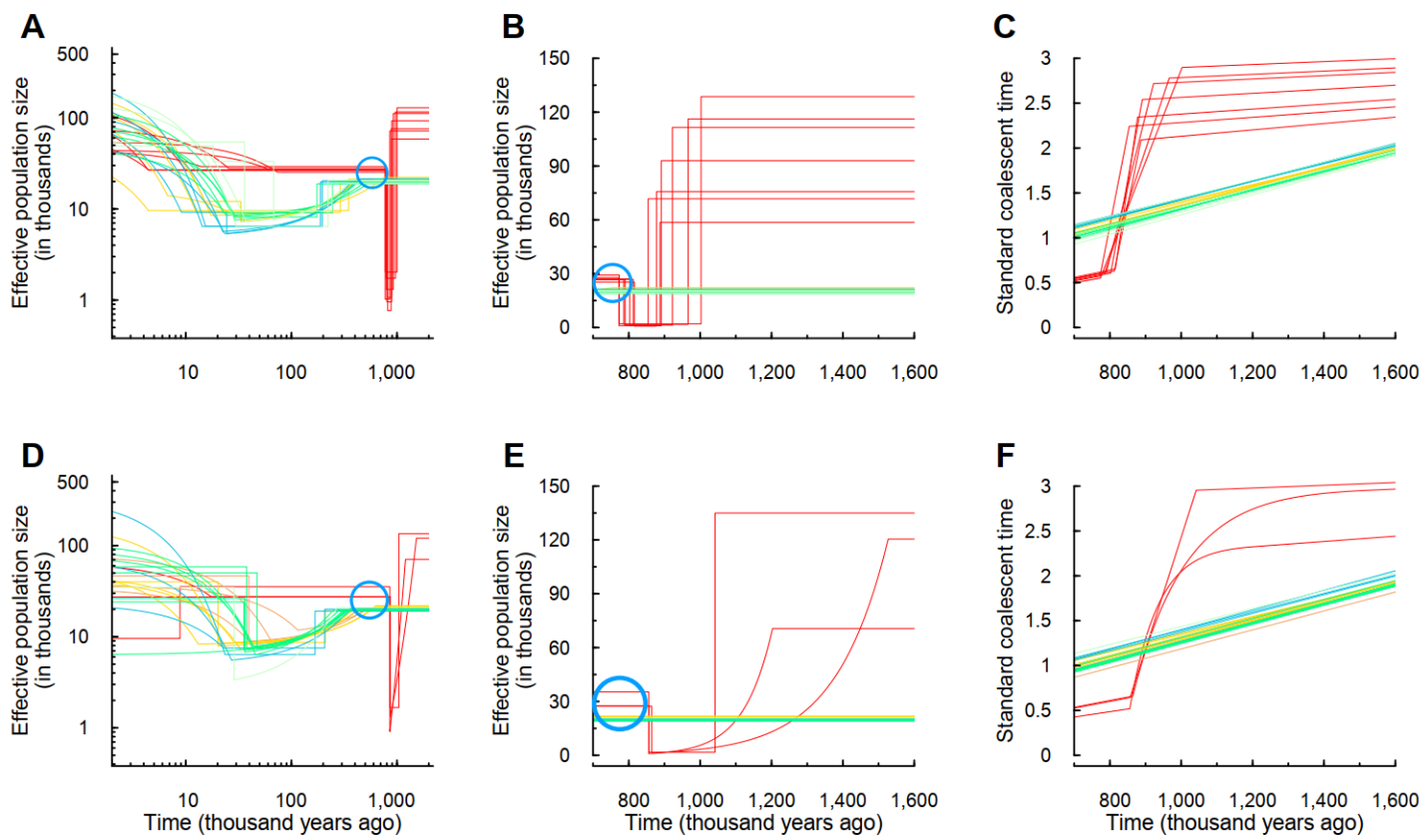
to be 24 years.

Figure 3. FitCoal estimated histories of human populations using 1000GP and

HGPD-CEPH genomic data sets. (A) Estimated histories of 26 populations in 1000GP. (B) Linear-scaled estimation of histories of 1000GP populations during the super bottleneck period. (C) Calendar time vs standard coalescent time of estimated histories of 1000GP populations. (D) Estimated histories of 24 HGPD-CEPH populations. (E) Linear-scaled estimation of histories of HGPD-CEPH populations during the super bottleneck period. (F) Calendar time $v s$ standard coalescent time of estimated histories of HGPD-CEPH populations. Various color lines indicate the following: red, African populations; yellow, European populations; brown, Middle East populations; blue, East Asian populations; green, Central or South Asian populations; and dark sea green, American populations. Blue circles show the population size gap between the African and non-African populations, indicating the hidden effect of the super bottleneck in non-African populations. The mutation rate is assumed to be $1.2 \times 10^{-8}$ per base per generation, and a generation time is assumed 

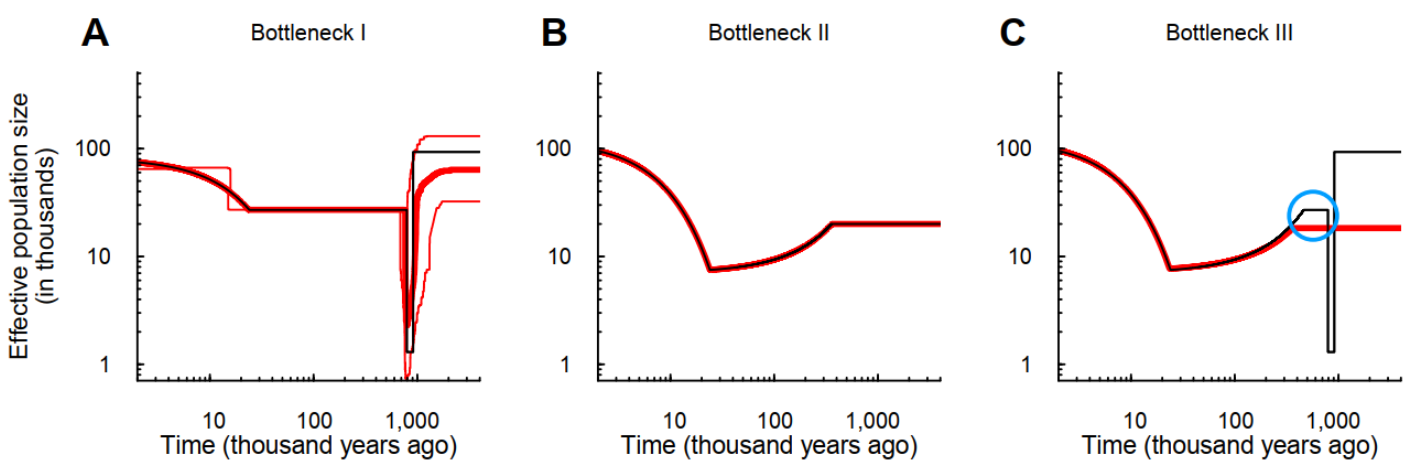

Figure 4. Verification of the super bottleneck. (A) Bottleneck I model, mimicking the demography of 1000GP African population and its estimated histories. (B) Bottleneck II model, mimicking the estimated demography of 1000GP non-African population and its estimated histories. (C) Bottleneck III model, mimicking the true demography of 1000GP non-African population and its estimated histories. Thin black lines indicate models. Thick red lines denote the medians of FitCoal estimated histories; thin red lines represent 2.5 and 97.5 percentiles of FitCoal estimated histories. Blue circle indicates the population size gap, the hidden effect of the super bottleneck in non-African populations. The mutation rate is assumed to be $1.2 \times$ $10^{-8}$ per base per generation, and a generation time is assumed to be 24 years. The number of simulated sequences is 202 in Bottleneck I and 200 in Bottleneck II and III. The length of simulated sequence is $800 \mathrm{Mb}$. 